\title{
Moment of Wave Energy Model in Water of Finite Depth
}

\author{
Pankaj Borah
}

\begin{abstract}
In this work, we have consider a particular wave energy device to create electrical energy in water. The device contains a submerged cylindrical obstacle placed above a plate which is also assume as a cylindrical structure. Also the diffracted potentials are derived by applying several mathematical model for each region of the fluid. Due to continuous flow of the fluid, we have introduce a system of equation for some unknown constants which are involve in potentials expression. Finally, we derived moment of the device due to diffraction wave field and present graphically.
\end{abstract}

Keywords : Diffraction, finite, device, wave number, moment.

\section{INTRODUCTION}

$\mathrm{T}$ he study of diffraction problem on floating obstacle can provide fundamental data of moment of the device, Many researchers have involved in this field, for example, in [2] discussed a scattering and radiation problem arising from wave interaction with a pair of cylinders and they presented the hydrodynamic properties of the structures due to both case of interaction. In [3] presented a mathematical method to determined the wave loads potentials on a vertical cylinder due to rotational as well as translation motion of the cylinder. In [4], [5] proposed a device consist a pair of cylinders and they analyzed the various effect on the forces occurs from diffracted wave field. In [6] developed three dimensional problem due to radiation by a submerged cylinder placed above a plate and they solved the problem by using the method of variables separation. In [7] studied a hydrodynamic problem occurs due to diffraction and radiation by a rectangular structure and they present the effect of still wall on the structure numerically. In [8] proposed a fundamental information regarding diffraction problem. In [9], [10], [11] considered only a single submerged cylinder I water and they solve the problem for both the diffraction and radiation..

\section{MATHEMATICAL FORMULATION}

\section{A. Scattering problem}

We assume a device consist of two cylindrical obstacle placed in a homogenous fluid of finite depth by taking $e_{1}$. The cylinders are also placed coaxially and also we assume that the radius of upper one i.e. submerged cylinder is less than the

Revised Manuscript Received on February 15, 2020.

* Correspondence Author

Pankaj Borah*, Mathematics department, North Eastern Regional Institute of Science and Technology, Itanagar, India-791109. Email: pankajborahmajuli@gmail.com radius of lower one i.e. plate. Let the radii of the upper one and lower one be $R_{s}$ and $R_{l}$, respectively. Let the Cartesian coordinate is $(x, y, z)$ which is the usual notation and the origin at $O$ as indicated in Fig. 1. Therefore the submerged cylinder i.e. upper one cylinder bound the fluid region is $r \leq R_{s}, 0 \leq \theta \leq 2 \pi,-e_{4} \leq z \leq-e_{5}$ and the plate i.e. lower one cylinder bound the fluid region is $r \leq R_{l}, 0 \leq \theta \leq 2 \pi,-e_{2} \leq z \leq-e_{3}$.

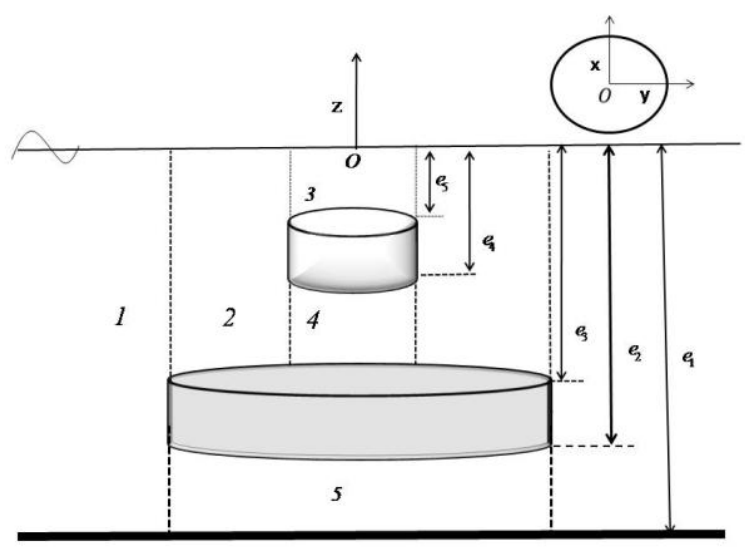

Fig. 1. Structural device

Since the diffracted wave field occurs based on theory of linear wave. Therefore the generalized potential $\phi(r, \theta, z, t)$ is given by $\phi(r, \theta, z, t)=\operatorname{Re}\left[\varphi(r, \theta, z) e^{-i \omega t}\right]$, where $r$ and $\theta$ stands for polar coordinate, Re[.] represents the real part and $\varphi(r, \theta, z)$ be the solution of Laplace's equation of three dimensional i.e. it is satisfies the equation in cylindrical form. Hence we have

$\frac{\partial^{2} \varphi}{\partial r^{2}}+\frac{1}{r} \frac{\partial \varphi}{\partial r}+\frac{1}{r^{2}} \frac{\partial^{2} \varphi}{\partial \theta^{2}}+\frac{\partial \varphi}{\partial z^{2}}=0$

\section{B. Governing equations}

The governing equation with boundary conditions are given by:

$$
\begin{aligned}
& \nabla^{2} \varphi_{s}=0 \\
& \frac{\partial \varphi_{s}}{\partial z}-\frac{\omega^{2}}{g} \varphi_{s}=0 \quad(z=0)
\end{aligned}
$$




$$
\begin{aligned}
& \frac{\partial \varphi_{s}}{\partial z}=0 \quad\left(z=-e_{1}\right) \\
& \frac{\partial \varphi_{s}}{\partial z}=0 \quad\left(z=-e_{3}, r<R_{l} ; z=-e_{5}, r<R_{s}\right) \\
& \frac{\partial\left(\varphi_{s}+\varphi_{i}\right)}{\partial r}=0 \quad\left(-e_{2}<z<-e_{3}, r=R_{l} ;-e_{4}<z<-e_{5}, r=R_{s}\right)
\end{aligned}
$$

and radiation condition is given by

$$
\lim _{r \rightarrow \infty} \sqrt{r}\left(\frac{\partial \varphi_{s}}{\partial r}-i k \varphi_{s}\right)=0
$$

Since the fluid domain can be divided into five subdomains viz. 1, 2, 3, 4 and 5, as indicated in Fig. 1 and hence the diffracted velocity potential for each region can be expressed as $\varphi_{s}{ }^{1}, \varphi_{s}{ }^{2}, \varphi_{s}{ }^{3}, \varphi_{s}{ }^{4}$ and $\varphi_{s}{ }^{5}$ respectively..

\section{MATCHING CONDITIONS}

To preserve the continuity fluid flow. along the boundary $r=R_{l}$ we have

$$
\begin{aligned}
& \varphi_{s}^{1}=\varphi_{s}^{2} \quad\left(-e_{3} \leq z \leq 0\right) \\
& \varphi_{s}^{1}=\varphi_{s}^{5} \quad\left(-e_{1} \leq z \leq-e_{2}\right) \\
& \frac{\partial \varphi_{s}^{1}}{\partial r}= \begin{cases}\frac{\partial \varphi_{s}^{2}}{\partial r} & \left(-e_{3} \leq z \leq 0\right) \\
-\frac{\partial \varphi_{i}}{\partial r} & \left(-e_{2} \leq z \leq-e_{3}\right) \\
\frac{\partial \varphi_{s}^{5}}{\partial r} & \left(-e_{1} \leq z \leq-e_{2}\right)\end{cases}
\end{aligned}
$$

Along the boundary $r=R_{s}$, we have

$$
\begin{gathered}
\varphi_{s}^{2}= \begin{cases}\varphi_{s}^{3} & \left(-e_{5} \leq z \leq 0\right) \\
\varphi_{s}^{4} & \left(-e_{3} \leq z \leq-e_{4}\right)\end{cases} \\
\frac{\partial \varphi_{s}^{2}}{\partial r}= \begin{cases}\frac{\partial \varphi_{s}^{3}}{\partial r} & \left(-e_{5} \leq z \leq 0\right) \\
\frac{\partial \varphi_{i}}{\partial r} & \left(-e_{4} \leq z \leq-e_{5}\right) \\
\frac{\partial \varphi_{s}^{4}}{\partial r} & \left(-e_{3} \leq z \leq-e_{4}\right)\end{cases}
\end{gathered}
$$

\section{SOLUTION TO THE PROBLEM}

The solution for the diffracted potential in each region are derived analytically by using the method of separation of variables

\section{- Region 1:}

$\varphi_{s}^{1}=\sum_{m=0}^{\infty} \sum_{n=0}^{\infty} A_{m, n} \frac{R_{m}\left(a_{n} r\right)}{R_{m}\left(a_{n} R_{l}\right)} \cos \left[a_{n}\left(z+e_{1}\right)\right] \cos m \theta$,

\section{- Region 2:}

$$
\begin{aligned}
\varphi_{s}^{2}=-\varphi_{i}+\sum_{m=0}^{\infty} \sum_{n=0}^{\infty}[ & {\left[B_{m, n} \frac{S_{m}\left(b_{n} r\right)}{S_{m}\left(b_{n} R_{s}\right)}+C_{m, n} \frac{T_{m}\left(b_{n} r\right)}{T_{m(}\left(b_{n} R_{s}\right)}\right] \times } \\
& \cos \left[b_{n}\left(z+e_{3}\right)\right] \cos m \theta,
\end{aligned}
$$

- Region 3: $\varphi_{s}^{3}=-\varphi_{i}+\sum_{m=0}^{\infty} \sum_{n=0}^{\infty} D_{m, n} U_{m}\left(c_{n} r\right) \cos \left[c_{n}\left(z+e_{5}\right)\right] \cos m \theta$,

- Region 4:

$\varphi_{s}^{4}=-\varphi_{i}+\sum_{m=0}^{\infty}\left[E_{m, 0} r^{m}+\sum_{n=1}^{\infty} E_{m, n} \frac{I_{m}\left(d_{n} r\right)}{I_{m}\left(d_{n} R_{s}\right)} \cos \left[d_{n}\left(z+e_{3}\right)\right]\right] \times$ $\cos m \theta$,

\section{- Region 5:}

$$
\varphi_{s}^{5}=-\varphi_{i}+\sum_{m=0}^{\infty}\left[F_{m, 0} r^{m}+\sum_{n=1}^{\infty} F_{m, n} \frac{I_{m}\left(\delta_{n} r\right)}{I_{m}\left(\delta_{n} R_{l}\right)} \cos \left[\delta_{n}\left(z+e_{1}\right)\right]\right] \times
$$
$\cos m \theta$,

where $A_{m, n}, B_{m, n}, C_{m, n}, D_{m, n}, E_{m, n}$ and $F_{m, n}$ are the unknown and $a_{n}, b_{n}, c_{n}, d_{n}$ and $\delta_{n}$ can be determined from the dispersion relation:

$\left\{\begin{array}{l}a_{n}=-i k \quad \omega^{2}=g k \tanh \left(k e_{1}\right), n=0 \\ \omega^{2}=-g a_{n} \tan \left(a_{n} e_{1}\right) \quad n=1,2, \ldots\end{array}\right.$

$\left\{\begin{array}{l}b_{n}=-i k_{1} \quad \omega^{2}=g k_{1} \tanh \left(k_{1} e_{3}\right), n=0 \\ \omega^{2}=-g b_{n} \tan \left(b_{n} e_{3}\right) \quad n=1,2, \ldots\end{array}\right.$

$\left\{c_{n}=-i k_{2} \quad \omega^{2}=g k_{2} \tanh \left(k e_{2}\right), n=0\right.$

$\omega^{2}=-g c_{n} \tan \left(c_{n} e_{2}\right) \quad n=1,2, \ldots$

$d_{n}=\frac{n \pi}{e_{3}-e_{1}} \quad n=0,1,2, \ldots$

$\delta_{n}=\frac{n \pi}{e_{1}-e_{2}} \quad n=0,1,2, \ldots$

The radial functions $R_{m}(),. S_{m}(),. T_{m}($.$) and U_{m}($.$) are$ given by

$R_{m}\left(a_{n} r\right)=H_{m}^{(1)}(k r)=H_{m}^{(1)}\left(i a_{0} r\right), \quad n=0$

$R_{m}\left(a_{n} r\right)=K_{m}\left(a_{n} r\right), \quad n=1,2, \ldots$

$S_{m}\left(b_{n} r\right)=H_{m}^{(1)}\left(k_{1} r\right), \quad n=0$

$S_{m}\left(b_{n} r\right)=K_{m}\left(b_{n} r\right), \quad n=1,2, \ldots$ 


$$
\begin{array}{ll}
T_{m}\left(b_{n} r\right)=I_{m}\left(b_{n} r\right), & n=1,2, \ldots \\
U_{m}\left(c_{n} r\right)=J_{m}\left(k_{2} r\right), & n=0 \\
U_{m}\left(c_{n} r\right)=I_{m}\left(c_{n} r\right), & n=1,2, \ldots
\end{array}
$$

\section{UNKNOWN COEFFICIENTS}

The unknown constants occurs in potentials expression are calculated as follow: (i) first we apply the equation into potentials expression (ii) we multiply both sides by a orthogonal eigenfunction. Therefore we have

$$
\begin{aligned}
& \left.\int_{-e_{3}}^{0} \varphi_{s}^{1}\right|_{r=R_{l}} \cdot \cos \left[b_{l}\left(z+e_{3}\right)\right] d z=\left.\int_{-e_{3}}^{0} \varphi_{s}^{2}\right|_{r=R_{s}} \cdot \cos \left[b_{l}\left(z+e_{3}\right)\right] d z \\
& \left.\int_{-e_{1}}^{-e_{2}} \varphi_{s}^{1}\right|_{r=R_{l}} \cdot \cos \left[c_{l}\left(z+e_{1}\right)\right] d z=\left.\int_{-e_{1}}^{-e_{2}} \varphi_{s}^{5}\right|_{r=R_{l}} \cdot \cos \left[c_{l}\left(z+e_{1}\right)\right] d z \\
& \left.\int_{-e_{1}}^{0} \frac{\partial \varphi_{s}^{1}}{\partial r}\right|_{r=R_{l}} \cdot \cos \left[b_{l}\left(z+e_{1}\right)\right] d z=\left.\int_{-e_{3}}^{0} \frac{\partial \varphi_{s}^{2}}{\partial r}\right|_{r=R_{l}} \cdot \cos \left[b_{l}\left(z+e_{1}\right)\right] d z- \\
& \left.\int_{-e_{1}}^{-e_{2}} \frac{\partial \varphi_{s}^{5}}{\partial r}\right|_{r=R_{l}} \cdot \cos \left[b_{l}\left(z+e_{1}\right)\right] d z+\left.\int_{-e_{1}}^{-} \frac{\partial \varphi_{s}^{5}}{\partial r}\right|_{r=R_{l}} \cdot \cos \left[b_{l}\left(z+e_{1}\right)\right] d z \\
& \left.\int_{-e_{5}}^{0} \varphi_{s}^{2}\right|_{r=R_{s}} \cdot \cos \left[c_{l}\left(z+e_{5}\right)\right] d z=\left.\int_{-e_{5}}^{0} \varphi_{s}^{3}\right|_{r=R_{s}} \cdot \cos \left[c_{l}\left(z+e_{5}\right)\right] d z \\
& \left.\int_{-e_{4}}^{-e_{3}} \frac{\left.\varphi_{s}^{2}\right|_{r=R_{s}} \cdot \cos \left[b_{l}\left(z+e_{3}\right)\right] d z=\left.\int_{-e_{3}}^{-e_{4}} \varphi_{s}^{4}\right|_{r=R_{s}} \cdot \cos \left[b_{l}\left(z+e_{3}\right)\right] d z}{\partial r}\right|_{r=R_{s}} \cdot \cos \left[b_{l}\left(z+e_{3}\right)\right] d z=\left.\int_{-e_{5}}^{0} \frac{\partial \varphi_{s}^{3}}{\partial r}\right|_{r=R_{s}} \cdot \cos \left[b_{l}\left(z+e_{3}\right)\right] d z \\
& \quad-\left.\int_{-e_{4}}^{-e_{5}} \frac{\partial \varphi_{i}}{\partial r}\right|_{r=R_{s}} \cdot \cos \left[b_{l}\left(z+e_{3}\right) d z+\left.\int_{-e_{3}}^{-e_{4}} \frac{\partial \varphi_{s}^{4}}{\partial r}\right|_{r=R_{s}} \cos \left[b_{l}\left(z+e_{3}\right) d z\right.\right.
\end{aligned}
$$

Let us assume

$$
\begin{aligned}
& L\left(x_{n}, y_{n}, A_{1}, A_{2}, k_{1}, k_{2}\right)=\int_{k_{1}}^{k_{2}} \cos \left[x_{n}\left(z+A_{1}\right)\right] \cdot \cos \left[y_{n}\left(z+A_{2}\right)\right] d z, \\
& N\left(x_{n}, A_{1}, k_{1}, k_{2}\right)=\int_{k_{1}}^{k_{2}} \cos ^{2}\left[x_{n}\left(z+A_{1}\right)\right] d z .
\end{aligned}
$$

Now using the above operator given by equations (37) and (38), we get

$$
\begin{aligned}
\sum_{n=0}^{\infty} A_{m, n} M\left(a_{n}, \alpha_{l}, e_{1}, e_{3},-e_{3}, 0\right)= & \frac{i g}{\omega} \frac{\varepsilon_{m} J_{m}\left(k R_{l}\right)}{\cosh \left(k e_{1}\right)} M\left(a_{0}, b_{l}, e_{1}, e_{3},-e_{3}, 0\right)+ \\
& {\left[B_{m, l} P_{m l}+C_{m, l} Q_{m l}\right] N\left(b_{l}, e_{3},-e_{3}, 0\right) }
\end{aligned}
$$

$$
\begin{aligned}
& \sum_{n=0}^{\infty} A_{m, n} M\left(a_{n}, \delta_{l}, e_{1}, e_{1},-e_{1},-e_{2}\right)=\frac{i g}{\omega} \frac{\varepsilon_{m} J_{m}\left(k R_{l}\right)}{\cosh \left(k e_{1}\right)} \times \\
& M\left(a_{0}, \delta_{l}, e_{1}, e_{1},-e_{1},-e_{2}\right)+F_{m, l} R_{m l}^{b} N\left(\delta_{l}, e_{1},-e_{1},-e_{2}\right)
\end{aligned}
$$

where

$$
\begin{aligned}
P_{m l} & =\frac{S_{m}\left(b_{l} R_{l}\right)}{S_{m}\left(b_{l} R_{s}\right)}, Q_{m l}=\frac{T_{m}\left(b_{l} R_{l}\right)}{T_{m}\left(b_{l} R_{s}\right)} . \\
R_{m l}^{b} & = \begin{cases}R_{b}^{m} & l=0 \\
1 & l=1,2, \ldots\end{cases} \\
V_{m n} & =\frac{b_{n} S_{m}^{\prime}\left(b_{n} R_{l}\right)}{S_{m}\left(b_{n} R_{s}\right)}, \quad W_{m n}=\frac{b_{n} T_{m}^{\prime}\left(b_{n} R_{l}\right)}{T_{m}\left(b_{n} R_{s}\right)}
\end{aligned}
$$

$$
\begin{gathered}
\sum_{n=0}^{\infty}\left(B_{m, n}+C_{m, n}\right) M\left(b_{n}, c_{l}, e_{3}, e_{2},-e_{2}, 0\right)=D_{m, l} \times \\
U\left(c_{l} R_{s}\right) N\left(c_{l}, e_{2},-e_{2}, 0\right)
\end{gathered}
$$

$$
N\left(c_{l}, e_{3},-e_{3},-e_{1}\right)
$$

$$
\begin{array}{r}
{\left[B_{m, l} G_{m l}+C_{m, l} H_{m l}\right] N\left(b_{l}, e_{3},-e_{3}, 0\right)=\sum_{n=0}^{\infty} D_{m, n} d_{n} U^{\prime}\left(d_{n} R_{s}\right) \times} \\
M\left(d_{n}, b_{l}, e_{2}, e_{3},-e_{2}, 0\right)+\sum_{n=0}^{\infty} E_{m, n} O_{m n} \times \\
M\left(c_{n}, b_{l}, e_{3}, e_{3},-e_{3},-e_{1}\right)
\end{array}
$$

$X_{m n}=\left\{\begin{array}{l}m R_{l}^{m-1} \quad n=0 \\ \frac{\delta_{n} I_{m}^{\prime}\left(\delta_{n} R_{l}\right)}{I_{m}\left(\delta_{n} R_{l}\right)} \quad n=1,2, \ldots\end{array}\right.$

$R_{m l}= \begin{cases}R_{s}^{m} & l=0 \\ 1 & l=1,2, \ldots\end{cases}$

$G_{m l}=\frac{b_{l} S_{m}^{\prime}\left(b_{l} R_{s}\right)}{S_{m}\left(b_{l} R_{s}\right)} \quad, \quad H_{m l}=\frac{b_{l} T_{m}^{\prime}\left(b_{l} R_{s}\right)}{T_{m}\left(b_{l} R_{s}\right)}$

$O_{m n}=\left\{\begin{array}{lc}m R^{m-1} & n=0 \\ \frac{c_{n} I_{m}^{\prime}\left(c_{n} R_{s}\right)}{I_{m}\left(c_{n} R_{s}\right)} & n=1,2, \ldots\end{array}\right.$ 


\section{Moment of Wave Energy Model in Water of Finite Depth}

Therefore the unknowns are given by the solution of above system of equation given by (33)-(34).

\section{MOMENT}

Since moment due to diffraction wave field generated by interaction with the obstacle in water is determined from the dynamic fluid pressure. Therefore the pressure can be obtained by using equation of continuous fluid flow given by Euler and it is given by

$p=-\rho \frac{\partial \phi_{s}}{\partial t}$.

And a simple surface integration carried out to determined the moment of the submerged cylinder i.e. upper cylinder and it can be obtained as follow:

$$
\begin{aligned}
M_{s}= & -i \rho \omega\left[\int_{0}^{2 \pi} \int_{-e_{4}}^{-e_{5}} \varphi_{s}^{2}\left(R_{s}, \theta, z\right) \cdot\left(z+e_{4}+e_{5}\right) \cdot R_{s} \cos \theta d z d \theta\right. \\
& -\int_{0}^{2 \pi} \int_{0}^{R_{s}} \varphi_{s}^{3}\left(r, \theta,-e_{5}\right) \cdot r^{2} \cos \theta d r d \theta+ \\
& \int_{0}^{2 \pi} \int_{0}^{R_{s}} \varphi_{s}^{4}\left(r, \theta,-e_{4}-e_{5}\right) \cdot r^{2} \cos \theta d r d \theta
\end{aligned}
$$

\section{RESULT AND DISCUSSION}

In this section, discussed the effect of various parameters on the moment due to diffraction by submerged cylinder. Therefore we fixed some parameters throughout the numerical presentation and these are taking as follow: $e_{1}=3 m, g=9.8 m / s^{2}, e_{3}=0.75 m$. Now we investigate the effect of radius of plate on the moment. In Fig (2), we plot the non-dimensional moment versus non-dimensional wave number for $R_{l}=0.2 e_{1}$ and we observed that a obvious oscillation near lower range of wave number and for higher wave number, the moment almost vanish. Also the oscillation gives local extreme values. Hence for the range of wave number 0 to 0.5 , the maximum value of moment is 0.16291 and for the range 0 to 1.5 the maximum value is 0.30959. Similarly, in Fig. (3) we plot moment for $R_{l}=0.4 e_{1}$ and we observed a small oscillation as well as local maximum of moment occurs. Next in Fig. (4), we plot moment with same parameters of above presentation with $R_{l}=0.6 e_{1}$. From the figure we have seen that the curves of moment is smooth and only one maximum point occurs. Those maximum is also occurs within small value of wave number. Therefore from the Figs (2). (3) and (3), we have observed that the value of moment oscillating and local maximum occurs only for lower value of radius $R_{l}$ due to effect of the plate.

\section{CONCLUSION}

In this work, have derived the moment due to diffraction of wave interaction with a submerged obstacle in water. A simple analytical mathematical model have been used to solve this diffraction problem and we obtained analytical result for potential of diffracted wave field. By applying these potentials, we derived analytical representation of moment of the submerged cylinder. Also we present the graphical result of moment for different radius of the obstacle. 
From the graphical representation, we conclude that the oscillation behavior of moment is high for lower value of $R_{s}$ which will help a engineer to design a model of wave energy.

\section{NOTATIONS}

$g$ : Acceleration due to gravitational force

$\varphi_{i} \quad:$ Incident potential

$\varphi_{s} \quad:$ Diffraction potential

$\rho \quad$ : Density of the fluid

$\omega \quad$ : Angular frequency

$p \quad$ : Dynamic Pressure

$J_{1}($.$) : Bessel function of first kind$

$I_{1}($.$) \quad : modified Bessel function of first kind$

$H_{1}^{(1)}($.$) : Hankel function of first kind$

$H_{1}^{(2)}($.$) : Hankel function of second kind$

$K_{1}($.$) : modified Bessel function of second kind.$

\section{REFERENCES}

1. M. Abramowitz and I. A. Stegun, "Handbook of Mathematical Functions," New York: Dover, 1965.

2. Y. H. Zheng, Y. M. Shen, Y. G. You, B. J. Wu and L. Rong, "Hydrodynamic properties of two vertical truncated cylinders in waves," Ocean Engineering, vol. 32, March 2005, pp. 241-271.

3. D. D. Bhatta and M. Rahman, "On scatering and radiation problem for a cylinder in water of finite depth," International Journal of Engineering Science, vol. 41(9), May 2003, pp. 931-967.

4. Y. M. Shen,Y. H. Zheng, Y. G. You, "On the Radiation and Diffraction of Linear Water Waves by a Rectangular Structure over a Sill: Part I. Infinite Domain of Finite Water Depth," Ocean Engineering, vol. 32, June 2005, pp. 1073-1097.

5. B. J. Wu, Y. H. Zheng, Y. G. You, X. Y. Sun and Y. Chen, "On Scattering and Radiation Problem for a Cylinder over a Cylindrical Barrier in Water of Finite Depth," Engineering Science, vol. 6(2), June 2004, pp. 48-55. [In Chinese]

6. J, Bhattacharjee and C. G. Soares, "Wave Interaction with a Floating Rectangular Box Near a Vertical Wall with Step Type Bottom Topography," Journal of Hydrodynamics, vol. 22 (5), October 2010, pp., 91-96.

7. S. C. Jiang, B. Teng, D. Z. Ning and Z. Lin, "An Analytical Solution of Wave Diffraction by a Submerged Vertical Cylinder," The Ocean Engineering, vol. 28 (3), January 2010, pp. 68-75. [In Chinese]

8. P. Borah and M. Bishwakarma, "Three Dimensional Radiated Water Wave with a Submerged Cylinder in Presence of Circular Plate" International Journal of Engineering and Advanced, vol. 9(1). Octtober 2019, pp. 6665-6670.

9. R. C. MacCamy and R. A. Fuchs, "Wave forces on piles: A diffraction theory," Technical Memo, No. 69. US Army Beach Erosion Board, December 1954, p. 17.

10. M. Rahma, "Water waves Waves: Relating Modern Theory to Advanced Engineering," Applications. Clarendon Press, Oxford, 1995.

11. B. J. Wu, Y. H. Zheng and Y. G. You, "Response Amplitude and Hydrodynamic Force for a Buoy over a Convex," Journal of Waterway, Port, Coastal, and Ocean Engineering, vol. 132(2), March 2006, pp. 97-105.

\section{AUTHORS PROFILE}

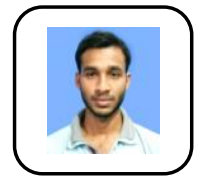

Pankaj Borah born in a small town of Assam, India. He got B.Sc, degree in Mathematics from a govt college Jorhat Science College under Dibrugarh University, Assam, India in 2014 and he was Mastered in Mathematical Science from Tezpur University, India Research scholar at NERIST, India. 